\title{
Mechanically ventilated COVID-19 patients failed to meet the criteria for the Berlin definition of ARDS
}

\author{
Denis Garot ${ }^{1} \cdot$ Lionel Tchatat Wangueu ${ }^{1,2} \cdot$ Charlotte Larrat $^{1,2} \cdot$ Antoine Guillon $^{1,2,3}$ (])
}

Received: 7 July 2021 / Accepted: 1 September 2021 / Published online: 14 September 2021

c) Springer-Verlag GmbH Germany, part of Springer Nature 2021

\section{Dear Editor,}

Using a consensus process, a panel of experts convened in 2011 have simplified and clarified the definition of acute respiratory distress syndrome (ARDS) and gave birth to the so called, Berlin definition of ARDS [1]. The Berlin definition was developed to achieve a more accurate definition and to better match clinical outcomes to severity of illness categories. It provided validated support for three strata of initial arterial hypoxaemia $(\mathrm{PaO} 2 / \mathrm{FiO} 2$ categories of $\leq 100$, 101-200, and 201-300 mm Hg), which correlated with mortality ( $45 \%, 32 \%$, and $27 \%$, respectively) [1]. Now, in 2020-21, critically ill COVID-19 patients presented typical morphological hallmarks of ARDS with lung edema, inflammation, and alveolar hemorrhage (i.e., diffuse alveolar damage) [2]. They were classified as ARDS, because the lung injury was due to an acute lung infection responsive of bilateral infiltrates on chest imaging and was responsive to arterial hypoxaemia despite mechanical ventilation with positive end-expiratory pressure and without cardiac failure explaining the respiratory failure. More than 3000 articles matched the keywords "COVID-19" and "ARDS" in 2020. However, we questioned whether ventilated COVID19 patients meet the Berlin definition of ARDS because of long latency periods between symptom onset and respiratory failure (should be 1 week or less in the definition of ARDS). To test this assumption, we reported the time from symptoms onset to hospitalization, ICU admission and initiation of the mechanical ventilation in critically ill patients due to community-acquired SARS-CoV-2 infection.

Antoine Guillon

antoine.guillon@univ-tours.fr

1 Intensive Care Unit, Tours University Hospital, 2 Bd Tonnellé, 37044 Tours Cedex 9, France

2 University of Tours, Tours, France

3 Research Center for Respiratory Diseases, INSERM U1100, Tours, France
In this single-center study (prospective recording from 2020-03-14 to 2020-04-12), we included critically ill patients due to community-acquired SARS-CoV-2 infection and receiving mechanical ventilation with partial pressure of arterial oxygen to fraction of inspired oxygen $\left(\mathrm{PaO}_{2} /\right.$ $\mathrm{FiO}_{2}$ ) of $300 \mathrm{~mm} \mathrm{Hg}$ or less (with positive end-expiratory pressure of $5 \mathrm{~cm} \mathrm{H}_{2} \mathrm{O}$ or more), with bilateral infiltrates on chest imaging and without cardiac failure explaining the respiratory failure. We reported patients 'characteristics and latency periods. No nominative, sensitive or personal data of patients have been collected. Our study involved the reuse of already recorded and anonymized data. The study falls within the scope of the French Reference Methodology MR-004, which require neither information nor consent of the included individuals. Results are presented in mean \pm SD.

We studied 191 patients of $64.8 \pm 10.4$ years old, mostly male (64.9\%), with $\mathrm{PaO}_{2} / \mathrm{FiO}_{2}$ of $141 \pm 57 \mathrm{~mm} \mathrm{Hg}$, treated with neuromuscular blockers $(98.2 \%)$ and prone position $(90.9 \%)$, and having in ICU mortality of $20.9 \%$. The mean times from symptoms onset to hospitalization, ICU admission and initiation of the mechanical ventilation were, respectively, $7.5 \pm 3.3,8.8 \pm 3.3$, and $10.5 \pm 4.0$ days (Fig. 1). Only $21.9 \%(42 / 191)$ of these critically ill and ventilated patients had respiratory failure developed within 1 week of the clinical onset and could actually be classified as ARDS according to the Berlin definition.

In our experience, the large majority of ventilated COVID-19 patients failed to meet the criteria for the Berlin definition of ARDS, despite severe lung injury and livethreatening condition. Critically ill patients due to SARSCoV-2 infection cannot be classified as sepsis [3] or as ARDS. The purpose of these observations is not to be picky with the definitions, but to highlight that COVID-19 has been a game changer in our ability to stratify the patients. Definition need to evolve as new information and experience is gained [4]. Considering an expanded definition of ARDS due to the COVID-19 pandemic is a legitimate question that 


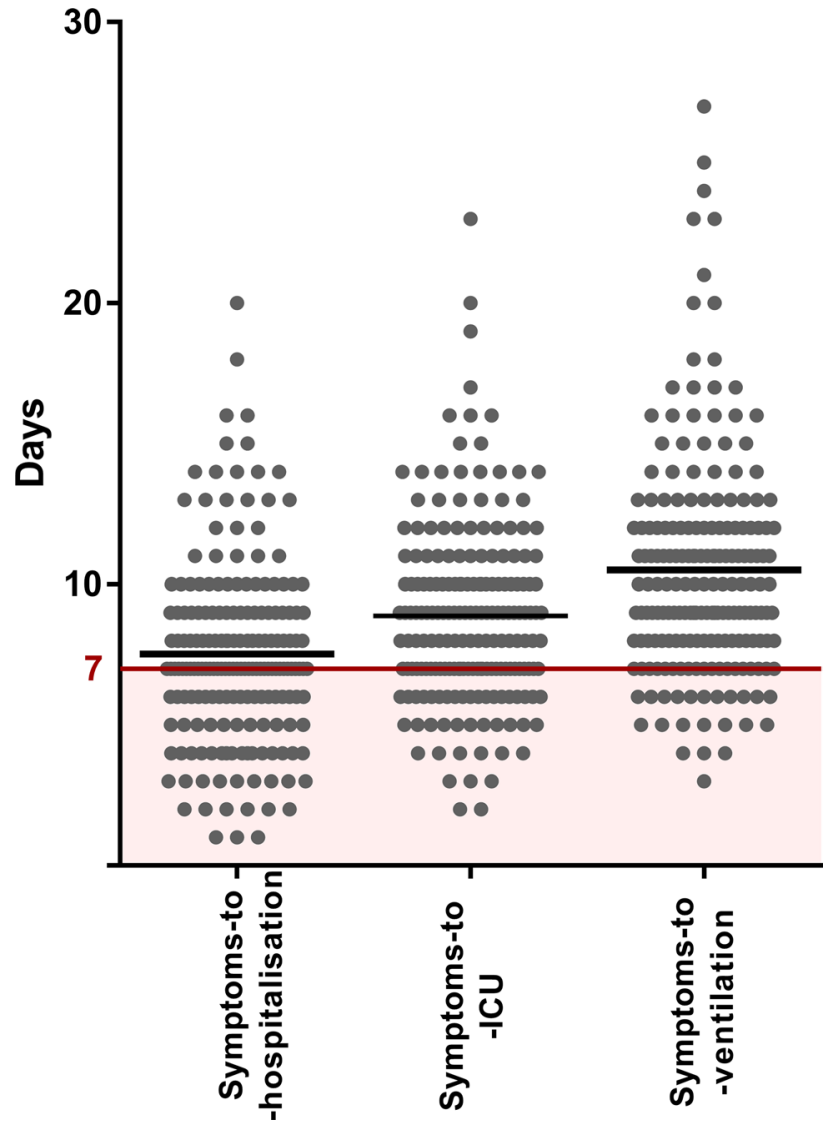

Fig. 1 Latency periods between symptom onset and respiratory failure of critically ill COVID-19 patients requiring invasive mechanical ventilation. Patients received invasive mechanical ventilation with $\mathrm{PaO}_{2} / \mathrm{FiO}_{2}$ of $300 \mathrm{~mm} \mathrm{Hg}$ or less, with bilateral infiltrates on chest imaging and without cardiac failure explaining the respiratory failure. Times from symptom onsets to hospitalization, ICU admission, and initiation of invasive mechanical ventilation are represented by dots (individual value) or black lines (mean value). The 1-week limit for the latency period of the Berlin definition of ARDS is represented by the red line

should be considered. Modifying the latency period for the inciting clinical disorder should be included in this discussion. Given the slower progression of respiratory failure in COVID-19 compared with other causes of ARDS, we propose that a revised definition of ARDS could consider latency longer than 7 days from identification of the ARDS risk factor. As already suggested [4] and in agreement with our results, a latency period (between symptom onset and respiratory failure) up to 14 days seems to be optimal to define COVID-19 ARDS. Whether this longer latency period may apply to all respiratory viral infections including influenza is a more complicated question. In our experience, the time between the onset of symptoms and hospitalization is inferior to one week for viral pneumonia (non-COVID-19; unpublished data from [5]).
In conclusion, we observed that the long latency period between symptom onset and respiratory failure preclude ventilated COVID-19 patients to be stratified as ARDS. We proposed to expand the latency period (up to 14 days) and welcome the feed-back of clinicians caring for critically ill COVID-19 patients.

Acknowledgements We thank all medical students who contributed to database implementation.

Author contributions AG and DG conceived and designed the study. DG performed the data retrieval. DG and AG have accessed and verified the underlying data. DG, LLT, CL and AG were involved in drafting the manuscript. DG, LLT, CL and AG read and approved the final version to be published.

Funding No funding was used for this study.

Data availability The datasets used and/or analyzed for this research letter are available from the corresponding author on reasonable request.

\section{Declarations}

Conflict of interests No competing interests to declare.

Ethics approval and consent to participate No nominative, sensitive or personal data of patients have been collected. Our study involved the reuse of already recorded and anonymized data. The study falls within the scope of the French Reference Methodology MR-004, which require neither information nor consent of the included individuals.

Consent for publication Not applicable.

\section{References}

1. ARDS Definition Task Force, Ranieri VM, Rubenfeld GD, et al. Acute respiratory distress syndrome: the Berlin definition. JAMA. 2012;307:2526-33. https://doi.org/10.1001/jama.2012.5669.

2. Katzenstein AL, Bloor CM, Leibow AA. Diffuse alveolar damage - the role of oxygen, shock, and related factors. A review. Am J Pathol. 1976;85:209-28.

3. Ferreira M, Blin T, Collercandy N, et al. Critically ill SARSCoV-2-infected patients are not stratified as sepsis by the qSOFA. Ann Intensive Care. 2020;10:43. https://doi.org/10.1186/ s13613-020-00664-w.

4. Matthay MA, Thompson BT, Ware LB. The Berlin definition of acute respiratory distress syndrome: should patients receiving high-flow nasal oxygen be included? Lancet Respir Med. 2021. https://doi.org/10.1016/S2213-2600(21)00105-3.

5. Lhommet C, Garot D, Grammatico-Guillon L, et al. Predicting the microbial cause of community-acquired pneumonia: can physicians or a data-driven method differentiate viral from bacterial pneumonia at patient presentation? BMC Pulm Med. 2020;20:62. https://doi.org/10.1186/s12890-020-1089-y. 